\title{
Caracterização de frutos de espécies de pimentas produzidas na região do Cariri paraibano
}

\section{Judieldo de Morais Lima' ${ }^{1}$, Fabrício Soares Moreira ${ }^{1}$, João Paulo de Sousa ${ }^{1}$, Francisca Maria Barbosa ${ }^{2}$, Azenate Campos Gomes ${ }^{3}$, Carina Seixas Maia Dornelas ${ }^{1}$, Arthur Ribeiro Barbosa ${ }^{1}$, Alecksandra Vieira de Lacerda ${ }^{1}$,*}

\footnotetext{
${ }^{1}$ Centro de Desenvolvimento Sustentável do Semiárido. Universidade Federal de Campina Grande. Sumé-PB (CEP 58540-000).

${ }^{2}$ Instituto de Pesquisa em Fármacos e Medicamentos (IPeFarM). Universidade Federal da Paraíba. João Pessoa, PB (CEP 58037-275).

${ }^{3}$ Programa de Pós-Graduação Produtos Naturais e Sintéticos Bioativos. Universidade Federal da Paraíba. João Pessoa-PB (CEP 58037-275). E-mail alecvieira@yahoo.com.br.
}

Resumo. A pesquisa teve como objetivo caracterizar parâmetros físicos de frutos e sementes de espécies e variedades doces e picantes de pimentas produzidas na região do Cariri Ocidental paraibano. Foram selecionados frutos de espécies e variedades doces e picantes de pimentas: pimenta-jalapenho (Capsicum annuum var. annuum L.), pimentão (Capsicum annuum L.), chapeu de bispo (Capsicum baccatum L. var. pendulum (Willd.) Eshbaugh), dedo de moça (Capsicum baccatum L. var. umbilicatum (Vell.) Hunz. \& Barboza), dulce larga (Capsicum frutescens L.). As plantas foram produzidas no viveiro de mudas do Laboratório de Ecologia e Botânica pertencente ao Centro de Desenvolvimento Sustentável do Semiárido da Universidade Federal de Campina Grande ( $7^{\circ} 39^{\prime} 34,84^{\prime \prime} \mathrm{S}$ e $36^{\circ} 53^{\prime} 35,96^{\prime \prime} \mathrm{W}$; $538 \mathrm{~m}$ de altitude). Foram coletados todos os frutos das plantas após três meses do plantio, que foram analisados quanto ao comprimento longitudinal, diâmetro transversal, massa fresca dos frutos e quantidade de sementes por fruto. Utilizou-se balança analítica com precisão de $0,001 \mathrm{~g}$ e paquímetro digital com precisão de $0,01 \mathrm{~mm}$. Os dados foram analisados em Planilha Eletrônica Excel ${ }^{\circledR} 2013$ da Microsoft. O número de sementes por fruto dentro da mesma espécie ou variedade mostrou alta variabilidade, mesmo quando relacionado ao tamanho do fruto. Quanto à massa fresca dos frutos, os maiores valores foram registrados para o pimentão, dulce larga e chapéu de bispo, que produziram também o maior quantitativo de sementes. De modo geral, os dados obtidos contribuíram com informações básicas para a caracterização de frutos e sementes dos diferentes tipos de pimentas estudadas, se constituindo num importante indicador da sua produtividade e desenvolvimento.
Recebido:

29/01/2018

Aceito:

22/04/2018

Publicado:

30/04/2018

Acesso aberto

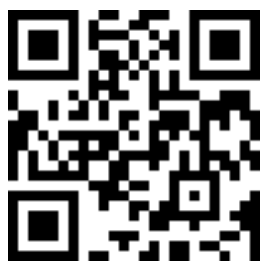

ORCID

(1) 0000-0002-3952-0316 Judieldo de Morais Lima

(D) 0000-0003-1822-5876

Fabrício Soares Moreira

D. 0000-0003-4783-7695

João Paulo de Sousa 
Palavras-chave: Biometria; Frutos; Sementes; Capsicum; Região semiárida.

\begin{abstract}
Characterization of fruits of pepper species produced in the Cariri region in Paraiba State, Brazil. This study aimed to characterize physical parameters of fruits and seeds of sweet and spicy peppers belonging to different species and varieties produced in the Cariri Ocidental region in Paraíba State, Brazil. Fruits of sweet and spicy peppers of the following species and varieties were selected: jalapeno pepper (Capsicum annuum var. annuum L.), bell pepper (Capsicum annuum L.), 'chapeu de bispo' (Capsicum baccatum L. var. pendulum (Willd.) Eshbaugh), 'dedo de moça' (Capsicum baccatum L. var. umbilicatum (Vell.) Hunz. \& Barboza), and 'dulce larga' (Capsicum frutescens L.). The plants were produced in the seedling nursery of the Ecology and Botany Laboratory of the Center for the Sustainable Development of the Semi-Arid Region at the Federal University of Campina Grande ( $7^{\circ} 39^{\prime} 34.84^{\prime \prime} \mathrm{S}$ and $36^{\circ} 53^{\prime} 35.96^{\prime \prime} \mathrm{W}$; $538 \mathrm{~m}$ of altitude). Three months after planting, all plant fruits were collected. Then, they were analyzed as for longitudinal length, transverse diameter, fresh fruit mass, and number of seeds per fruit. An analytical scale with an accuracy of $0.001 \mathrm{~g}$ and a digital caliper with an accuracy of $0.01 \mathrm{~mm}$ were used. The data were analyzed in a Microsoft Excel $^{\circledR} 2013$ Spreadsheet. The number of seeds per fruit within the same species or variety showed high variability, even when it was related to fruit size. Regarding the fresh fruit mass, the highest values were recorded for bell pepper, 'dulce larga', and 'chapéu de bispo', which also produced the highest number of seeds. In general, the data obtained in this study contributed with basic information for the characterization of fruits and seeds of the different types of peppers studied here, constituting an important indicator of their productivity and development.
\end{abstract}

Keywords: Biometrics; Fruits; Seeds; Capsicum; Semi-arid region.
0000-0002-6273-0979
Francisca Maria
Barbosa

(ㄷ) 0000-0001-6184-3114

Azenate Campos

Gomes

(D) 0000-0003-0602-7979

Carina Seixas Maia

Dornelas

(1) 0000-0002-3435-3854

Arthur Ribeiro

Barbosa

(D) 0000-0002-9703-3997

Alecksandra Vieira de Lacerda

\section{Introdução}

As espécies de pimentas cujo gênero é definido como Capsicum pertencem à Família Solanaceae. Assim como o tomate e a berinjela, existem aproximadamente 25 espécies deste gênero (Pinheiro et al., 2012). Algumas espécies do gênero Capsicum foram descobertas com a chegada dos navegadores portugueses e espanhóis ao continente americano, as quais já eram cultivadas pelos nativos (EMBRAPA, 2006).

Este gênero é utilizado na culinária, nas crenças, na medicina alopática ou natural e inclusive como arma de defesa. A pimenta possui propriedades farmacológicas, produzindo remédios à base de capsaicina, composto orgânico presente no fruto (Pinto et al., 2013). 0 formato do fruto varia entre as espécies e o tipo de cultivar (doce ou picante), existindo frutos alongados, arredondados, triangulares ou cônicos, campanulados, quadrados ou retangulares.

Segundo Silva et al. (2011) tem-se que nos últimos anos, a produção de pimentas cresceu significativamente e são utilizadas como condimento, nas indústrias farmacêutica e bélica e ainda como plantas ornamentais. Devido as suas potencialidades são muito usadas na culinária por serem importantes fontes 
de três antioxidantes naturais, as vitaminas $\mathrm{C}$ e $\mathrm{E}$ e os carotenoides (Ribeiro et al., 2008).

O cultivo de pimentas ocorre praticamente em todas as regiões do país e é um dos melhores exemplos de agricultura familiar e de integração pequeno agricultor-agroindústria. As áreas cultivadas anualmente equivalem a cerca de dois mil hectares na região Centro-Oeste e nos estados de São Paulo e Ceará (Reifschneider, 2000).

Capsicum é uma planta de clima quente, sensível a baixas temperaturas e intolerante a geadas, com faixa de ocorrência entre $21{ }^{\circ} \mathrm{C}$ a $30^{\circ} \mathrm{C}$, sendo a média das mínimas ideal $18{ }^{\circ} \mathrm{C}$ e das máximas em torno de $35{ }^{\circ} \mathrm{C}$, o que favorece a germinação, frutificação, e o desenvolvimento de um produto de alto valor comercial com menor custo de produção (EMBRAPA, 2006). Neste sentido, o estado da Paraíba com suas características naturais pode se tornar um grande produtor de pimentas.

Moreira (1989) coloca que devido a sua posição geográfica as áreas da Paraíba são submetidas a forte exposição dos raios solares, com cerca de 3.000 horas anuais de insolação. Como se encontra em baixa latitude, a inclinação dos raios solares é menor, sendo maior a concentração destes raios por unidade de área, o que resulta em altas temperaturas durante todo o ano (Ayoade, 2002). 0 alto índice de insolação na região contribui assim para que as temperaturas sejam sempre altas, com média térmica anual em torno dos $25^{\circ} \mathrm{C}$ (SUDENE, 1990).

Assim, considerando que as altas temperaturas durante a maior parte do ano na região propiciam o clima ideal para cultivar e diante da importância socioeconômica das espécies de pimenta (Capsicum), o estudo objetivou caracterizar parâmetros físicos de frutos e sementes de espécies das variedades doces e picantes de pimentas produzidas na região do Cariri Ocidental paraibano.

\section{Material e métodos}

Foram selecionadas espécies das variedades doces e picantes de pimentajalapenho (Capsicum annuum var. annuum L.), pimentão (Capsicum annuum L.), chapéu de bispo (Capsicum baccatum L. var. pendulum (Willd.) Eshbaugh), dedo de moça (Capsicum baccatum L. var. umbilicatum (Vell.) Hunz. \& Barboza), dulce larga (Capsicum frutescens L.). Estas espécies foram produzidas no Viveiro do Laboratório de Ecologia e Botânica pertencente ao Centro de Desenvolvimento Sustentável do Semiárido $\left(7^{\circ} 39^{\prime} 34,84^{\prime \prime}\right.$ S e $36^{\circ} 53^{\prime} 35,96^{\prime \prime}$ W; $538 \mathrm{~m}$ de altitude) (Figura 1).

A produção vegetal ocorreu em sementeira e o substrato utilizado foi composto de adubo bovino, areia e barro na proporção 1:1:1. Após a emergência esperou-se um mês para o transplantio que foi realizado para vasos de $16,50 \mathrm{~cm}$ de largura e $25 \mathrm{~cm}$ de altura agrupadas no canteiro $1 \times 4 \mathrm{~m}$ e regadas duas vezes ao dia. A coleta dos frutos ocorreu três meses após o plantio, seguindo 0 seguinte quantitativo de produção por espécie: pimenta-jalapenho ( $C$. annuum var. annuum) - 15 frutos; pimentão (C. annuum) - sete frutos; chapeu de bispo (C. baccatum var. pendulum) - sete frutos; dedo de moça (C. baccatum var. umbilicatum) - 31 frutos; dulce larga (C. frutescens) - sete frutos.

Após a coleta dos frutos das variedades doce e picantes, as pimentas foram conduzidas para o Laboratório de Ecologia e Botânica (LAEB/CDSA/UFCG), onde foram beneficiadas. Os frutos foram analisados quanto ao comprimento longitudinal, diâmetro transversal, massa fresca frutos e quantidade de sementes por fruto. Utilizou-se balança analítica de precisão $(0,001 \mathrm{~g})$ e paquímetro digital com 0,01 mm. Os dados foram analisados em Planilha Eletrônica Excel ${ }^{\circledR} 2013$ da Microsoft. 


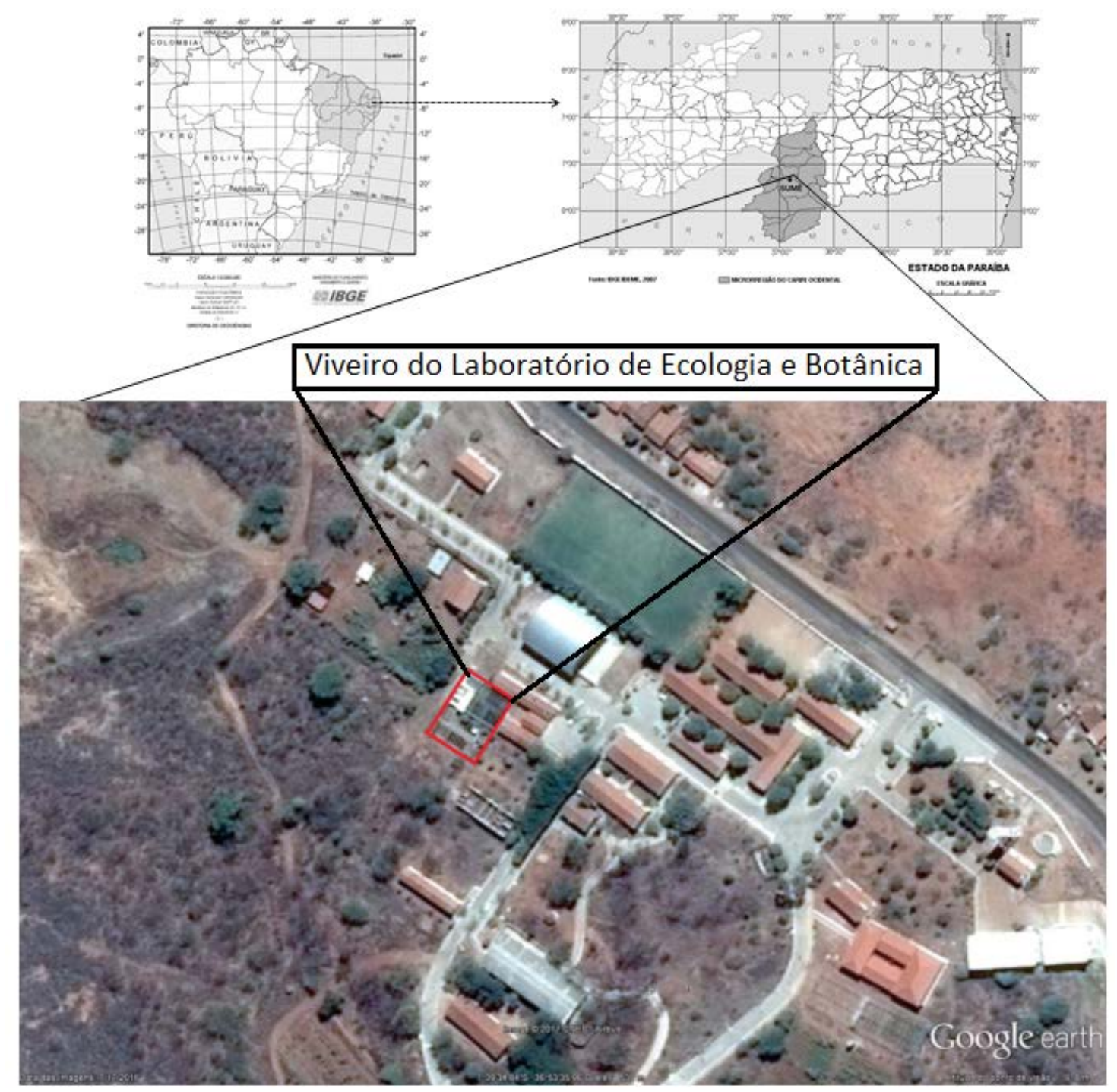

Figura 1. Localização do viveiro do Laboratório de Ecologia e Botânica, pertencente ao Centro de Desenvolvimento Sustentável do Semiárido, no Município de Sumé, Cariri Ocidental paraibano (7³9'36,56" S e $36^{\circ} 53^{\prime} 33,21^{\prime \prime}$ W; 540 m de altitude)

\section{Resultados e discussão}

Os dados obtidos para as espécies das variedades doces e picantes de pimentas encontram-se descritos na Tabela 1. Relacionado aos frutos da pimenta-jalapenho (C. annuum var. annuum), o comprimento variou de 3,1 a
5,2 cm, o diâmetro de 1 a $2 \mathrm{~cm}$, a quantidade de sementes por fruto de 6 a 140 e a massa total por fruto de 2,9 a 7,63 g. Para o pimentão (C. annuum) o comprimento variou de 3,7 a $6,4 \mathrm{~cm}$, o diâmetro de 2,8 a $5,3 \mathrm{~cm}$, a quantidade de sementes por fruto de 24 a 305 e a massa total por fruto de $12,36 \mathrm{a} 48,81 \mathrm{~g}$. 
Tabela 1. Caracterização dos frutos de espécies de pimentas produzidas no viveiro do Laboratório de Ecologia e Botânica (LAEB/CDSA/UFCG).

\begin{tabular}{|c|c|c|c|c|}
\hline $\begin{array}{l}\text { Fruto - } \\
\text { Número }\end{array}$ & Comprimento $(\mathrm{cm})$ & Diâmetro (cm) & Quantidade de Sementes & Peso total (g) \\
\hline \multicolumn{5}{|c|}{ Pimenta-jalapenho (C. annuum var. annuum) } \\
\hline 1 & 5,2 & 1,7 & 100 & 6,65 \\
\hline 2 & 4,7 & 1,9 & 109 & 7,01 \\
\hline 3 & 4,7 & 1,8 & 92 & 7,06 \\
\hline 4 & 4,0 & 2,0 & 101 & 7,21 \\
\hline 5 & 4,5 & 2,0 & 125 & 6,65 \\
\hline 6 & 4,0 & 1,1 & 85 & 4,90 \\
\hline 7 & 3,9 & 1,7 & 54 & 5,58 \\
\hline 8 & 3,8 & 1,7 & 140 & 7,63 \\
\hline 9 & 4,0 & 1,6 & 77 & 6,38 \\
\hline 10 & 3,7 & 1,0 & 6 & 2,90 \\
\hline 11 & 3,2 & 1,6 & 50 & 5,25 \\
\hline 12 & 3,9 & 1,2 & 8 & 4,04 \\
\hline 13 & 3,1 & 1,5 & 67 & 4,40 \\
\hline 14 & 3,8 & 1,7 & 69 & 6,08 \\
\hline 15 & 3,2 & 1,2 & 33 & 3,54 \\
\hline \multicolumn{5}{|c|}{ Pimentão (C. annuum) } \\
\hline 1 & 6,3 & 4,1 & 141 & 35,72 \\
\hline 2 & 5,7 & 5,3 & 305 & 48,81 \\
\hline 3 & 6,4 & 4,7 & 255 & 39,30 \\
\hline 4 & 4,3 & 3,2 & 169 & 15,56 \\
\hline 5 & 4,5 & 2,8 & 175 & 14,75 \\
\hline 6 & 4,0 & 3,0 & 24 & 12,36 \\
\hline 7 & 3,7 & 3,4 & 77 & 13,50 \\
\hline $\begin{array}{l}\text { Fruto - } \\
\text { Número }\end{array}$ & Comprimento $(\mathrm{cm})$ & Diâmetro (cm) & Quantidade de Sementes & Peso total (g) \\
\hline \multicolumn{5}{|c|}{ Chapéu de bispo (C. baccatum var. pendulum) } \\
\hline 1 & 2,9 & 4,5 & 104 & 13,87 \\
\hline 2 & 2,8 & 3,9 & 100 & 12,08 \\
\hline 3 & 2,8 & 3,6 & 106 & 15,54 \\
\hline 4 & 2,8 & 3,2 & 30 & 8,86 \\
\hline 5 & 2,2 & 3,8 & 33 & 7,16 \\
\hline 6 & 2,1 & 2,8 & 15 & 5,43 \\
\hline 7 & 1,8 & 2,8 & 55 & 4,81 \\
\hline \multicolumn{5}{|c|}{ Dedo de moça (C. baccatum var. umbilicatum) } \\
\hline 1 & 7,5 & 1,5 & 87 & 5,10 \\
\hline 2 & 7,0 & 1,4 & 77 & 4,47 \\
\hline 3 & 7,1 & 1,3 & 64 & 5,94 \\
\hline 4 & 7,1 & 1,5 & 88 & 6,28 \\
\hline 5 & 7,3 & 1,8 & 59 & 5,99 \\
\hline 6 & 6,9 & 1,6 & 88 & 5,84 \\
\hline 7 & 6,2 & 1,7 & 83 & 4,73 \\
\hline 8 & 7,3 & 1,6 & 69 & 5,87 \\
\hline 9 & 5,9 & 1,6 & 76 & 5,43 \\
\hline 10 & 6,4 & 1,3 & 64 & 3,30 \\
\hline 11 & 6,1 & 1,3 & 81 & 3,99 \\
\hline 12 & 6,1 & 1,5 & 23 & 3,74 \\
\hline 13 & 5,7 & 1,5 & 36 & 4,50 \\
\hline 14 & 5,8 & 1,6 & 57 & 3,66 \\
\hline 15 & 6,1 & 1,7 & 112 & 5,63 \\
\hline 16 & 5,5 & 1,6 & 91 & 4,11 \\
\hline
\end{tabular}


Tabela 1. Continuação.

\begin{tabular}{|c|c|c|c|c|}
\hline $\begin{array}{c}\text { Fruto - } \\
\text { Número }\end{array}$ & Comprimento (cm) & Diâmetro (cm) & Quantidade de Sementes & Peso total (g) \\
\hline \multicolumn{5}{|c|}{ Dedo de moça (C. baccatum var. umbilicatum) } \\
\hline 17 & 5,6 & 1,4 & 54 & 3,71 \\
\hline 18 & 5,5 & 1,5 & 85 & 3,96 \\
\hline 19 & 5,9 & 1,8 & 83 & 4,93 \\
\hline 20 & 6,9 & 1,8 & 57 & 4,72 \\
\hline 21 & 5,5 & 1,4 & 75 & 4,87 \\
\hline 22 & 4,8 & 1,4 & 74 & 3,40 \\
\hline 23 & 5,6 & 1,5 & 70 & 4,10 \\
\hline 24 & 5,8 & 1,7 & 66 & 4,34 \\
\hline 25 & 5,1 & 1,2 & 27 & 2,31 \\
\hline 26 & 4,5 & 1,3 & 92 & 3,07 \\
\hline 27 & 4,7 & 1,3 & 61 & 2,76 \\
\hline 28 & 5,0 & 1,4 & 53 & 2,60 \\
\hline 29 & 4,8 & 1,3 & 36 & 2,50 \\
\hline 30 & 4,2 & 1,8 & 12 & 3,14 \\
\hline 31 & 3,7 & 1,3 & 5 & 1,75 \\
\hline \multicolumn{5}{|c|}{ Dulce larga (C. frutescens) } \\
\hline 1 & 8,6 & 3,3 & 195 & 24,95 \\
\hline 2 & 8,9 & 3,0 & 218 & 17,50 \\
\hline $\begin{array}{c}\text { Fruto - } \\
\text { Número }\end{array}$ & Comprimento $(\mathrm{cm})$ & Diâmetro (cm) & Quantidade de Sementes & Peso total (g) \\
\hline 3 & 9,1 & 2,6 & 137 & 14,60 \\
\hline 4 & 9,3 & 2,2 & 136 & 12,75 \\
\hline 5 & 7,8 & 2,3 & 92 & 10,52 \\
\hline 6 & 7,2 & 2,9 & 184 & 13,47 \\
\hline 7 & 7,0 & 2,5 & 78 & 9,49 \\
\hline
\end{tabular}

Relacionado ao chapéu de bispo (C. baccatum var. pendulum) observou-se que o comprimento dos frutos variou de 1,8 a 2,9 cm, o diâmetro de 2,8 a $4,5 \mathrm{~cm}$, a quantidade de sementes por fruto de $15 \mathrm{a}$ 106 e a massa total por fruto de 4,81 a 15,54 g. Já a pimenta dedo de moça (C. baccatum var. umbilicatum), apresentou comprimento dos frutos com variações de 6,2 a 7,5 cm, diâmetro de 1,3 a 1,8 cm, a quantidade de sementes por fruto de 59 a 88 e massa total por fruto de 4,47 a 6,28 g.

Dulce larga (C. frutescens) obteve como valores e variações os seguintes resultados: altura variando de 7,0 a $9,3 \mathrm{~cm}$, a largura de 2,2 a $3,3 \mathrm{~cm}$, a quantidade de sementes por fruto de 78 a 218 e o peso total por fruto de 9,49 a $24,95 \mathrm{~g}$.

0 número de sementes por frutos dentro da mesma espécie ou variedade apresentou alta variabilidade, mesmo quando relacionado ao tamanho do fruto.
Freitas et al. (2009) afirmam que fatores, como o número de óvulos produzidos, quantidade e qualidade do pólen transferido, quantidade de nutrientes e fotoassimilados disponíveis para os frutos e sementes, ataques de herbívoros, predadores e doenças, são possíveis causas nas variações do número de sementes por fruto.

De modo geral, a variação nos valores dos dados biométricos pode ocorrer em virtude de alterações fisiológicas que provocam acréscimos nos valores das variáveis biométricas com o decorrer da maturação dos frutos (Abud, 2013).

Relacionado aos dados biométricos dos frutos de C. frutescens, esses corroboram com o trabalho de Carvalho (2014), que também relatou que essa espécie apresenta variabilidade morfológica expressa na forma, tamanho 
e cor de frutos inferior à verificada em outras espécies domesticadas do gênero, como as apresentadas na Tabela 1, possuindo assim, uma maior uniformidade dos frutos.

A pimenta dulce larga (C. frutescens) se destacou com os maiores valores de comprimento de frutos e o pimentão (C. annuum) obteve os maiores resultados em diâmetro, quantidade de sementes e peso total. Considerando à massa dos frutos frescos, os maiores valores foram registrados para o pimentão, dulce larga e chapéu de bispo, os quais produziram também o maior quantitativo de sementes (Figura 2).

A caracterização biométrica de frutos e sementes tem importância para a taxonomia, na identificação de variedades, para verificar a ocorrência de variações fenotípicas e nas associações com os fatores ambientais e genéticos (Pinto et al., 2003). Contribuindo também, para a avaliação da variabilidade genética entre cultivares para objetivo de conservação de recursos genéticos que é útil para identificar se dois indivíduos com fenótipos semelhantes mostram uma combinação gênica similar (Lefebvre et al., 2001).

De acordo com Sudré et al. (2005) assim como os marcadores moleculares os descritores fenotípicos têm sido um dos métodos bastante utilizados em estudos de medições de distância genética, sendo também sugerido em estudos de diversidade das espécies de pimentas e pimentões.

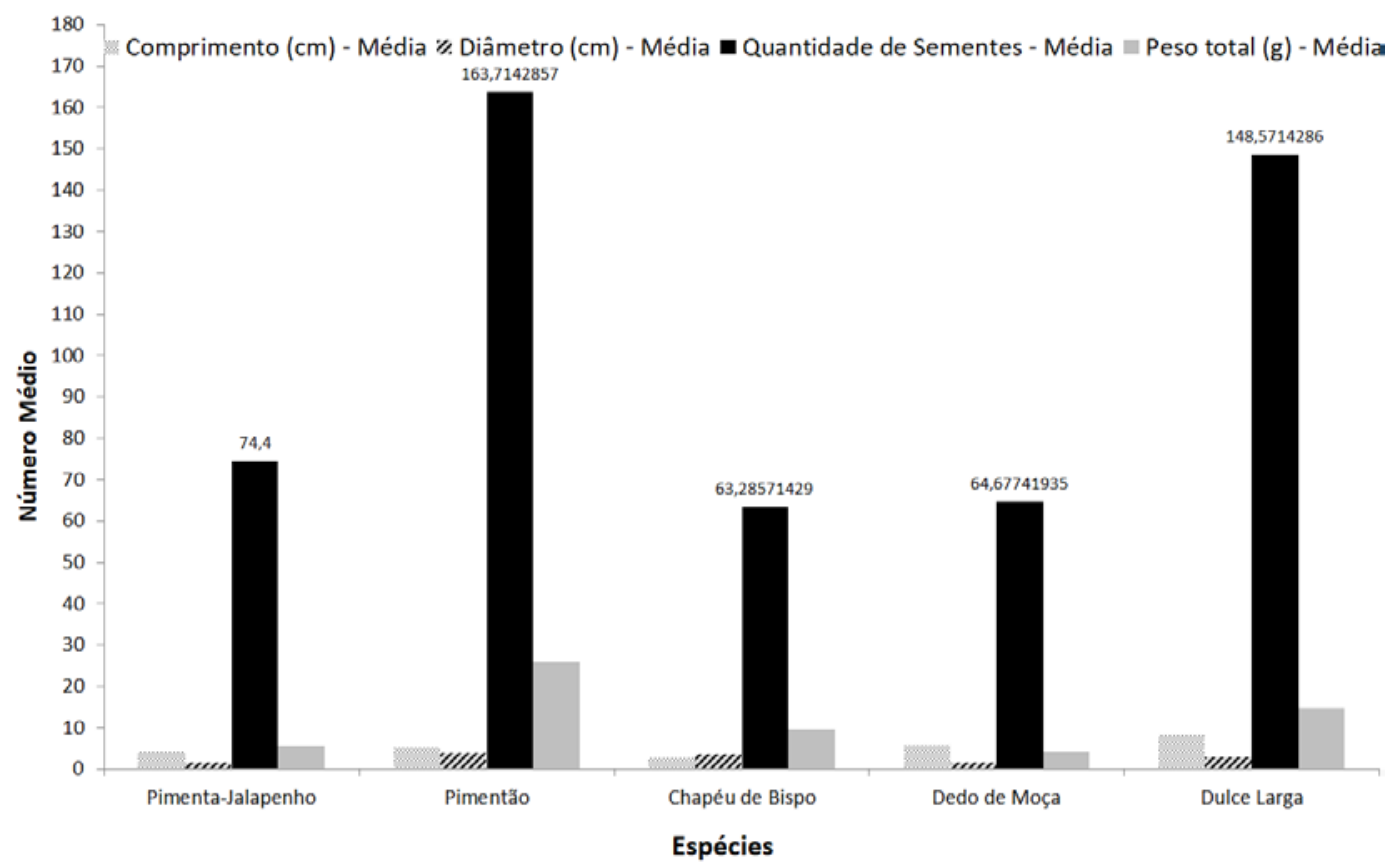

Figura 2. Dados médios dos parâmetros analisados dos frutos de espécies de pimentas produzidas no viveiro do Laboratório de Ecologia e Botânica (CDSA/UFCG). Pimenta-jalapenho (C. annuum var. annuum), pimentão (C. annuum), chapéu de bispo (C. baccatum var. pendulum ), dedo de moça (C. baccatum var. umbilicatum), dulce larga (C. frutescens).

\section{Conclusão}

Portanto, a pesquisa forneceu dados iniciais da caracterização de frutos e sementes de cinco tipo de pimentas, das quais o pimentão (C. annuum) e a pimenta dulce larga (C. frutescens) se destacaram em termos biométricos e de produtividade de semente por fruto, sendo C.annuum representante de 
pimentas doce e C.frutescens de pimentas picantes.

0 conjunto de dados apresentados fornece informações fundamentais para a caracterização de frutos e sementes dos diferentes tipos de pimentas estudadas, que são importantes indicadores da sua produtividade e desenvolvimento tanto em nível específico como em nível de variedades.

\section{Agradecimentos}

Ao Laboratório de Ecologia e Botânica do Centro de Desenvolvimento Sustentável do Semiárido, da Universidade Federal de Campina Grande, pela disponibilidade de seus espaços e estrutura para realização da pesquisa.

\section{Declaração de conflito de Interesses}

Os autores declaram não haver conflito de interesses.

\section{Referências}

Abud, H. F.; Araujo, E. F.; Araujo, R. F.; Araujo, A. V.; Pinto, C. M. F. Qualidade fisiológica de sementes das pimentas malagueta e biquinho durante a ontogênese. Pesquisa Agropecuária Brasileira, v. 48, p. 15461554, 2013. https://doi.org/10.1590/S0100204X2013001200003

Ayoade, J. O. Introdução à Climatologia para os trópicos. 8. ed. Rio de Janeiro: Bertrand Brasil, 2002.

Carvalho, S. I. C. Estudos filogenéticos e de diversidade em Capsicum e sua aplicação na conservação e uso de recursos genéticos das espécies $C$. frutescens e C. chinense. Brasília: Universidade de Brasília, 2014. (Tese de Doutorado).

EMBRAPA - Empresa Brasileira de Pesquisa Agropecuária. Pimentas do Gênero Capsicum no Brasil. Brasília: Embrapa, 2006.

Freitas, V. L. O.; Alves, T. H. S.; Lopes, R. M. F.; Lemos Filho, J.P. Biometria de frutos e sementes e germinação de sementes de Dimorphandra mollis Benth. e Dimorphandra
Wilsonii Rizz. (Fabaceae - Caesalpinioideae). Scientia Forestalis, v. 37, n. 81, p. 27-35, 2009. Disponível em: <http://www.ipef.br/ publicacoes/scientia/nr81/cap03.pdf>.

Acesso em: 26 ago. 2017.

Lefebvre, V.; Goffinet, B.; Chauvet, J. C.; Caromel, B.; Signoret P.; Brand R.; Palloix, A. Evaluation of genetic distances between pepper inbred lines for cultivar protection purposes: comparison of AFLP, RAPD and phenotypic data. Theoretical and Applied Genetics, v. 102, p. 741-750, 2001. https://doi.org/10.1007/s001220051705

Moreira, E. R. F. (Org.). Mesorregiões e microrregiões da Paraíba: delimitação e caracterização. João Pessoa: GAPLAN, 1988.

Pinheiro, J. B.; Amaro, G. B.; Pereira, R. B. Nematoides em pimentas do gênero Capsicum. Brasília: Embrapa, 2012. (Circular Técnica, 104).

Pinto, C. M. F.; Pinto, C. L. O.; Donzeles, S. M. L. Pimenta Capsicum: propriedades químicas, nutricionais, farmacológicas e medicinais e seu potencial para o agronegócio. Revista Brasileira de Agropecuária Sustentável, v. 3, p. 108-120, 2013. https://doi.org/ 10.21206/rbas.v3i2.225

Pinto, W. S.; Dantas, A. C. V. L.; Fonseca, A. A. O.; Ledo, C. A. S.; Jesus, S. C.; Calafange, P. L. P.; Andrade, E. M. Caracterização física, físico-química e química de frutos de genótipos de cajazeiras. Pesquisa Agropecuária Brasileira, v. 38, n. 9, p. 10591066, 2003. https://doi.org/10.1590/S0100204X2003000900006

Reifschneider, F. J. B. (Org). Capsicum: pimentas e pimentões do Brasil: Embrapa Hortaliças. Brasília: Embrapa, 2000.

Ribeiro, S. M. R.; Abranches, M. V.; Diniz, D. R.; Espeschit, A. C. R.; Peluzio, M. C. G.; Queiroz, J. H. Efeitos pró e antioxidantes dose-dependente de compostos bioativos. In: Costa, N. M. B.; Rosa, C. O. (Org.). Alimentos funcionais: benéficos para a saúde. Viçosa: Editora UFV, 2008. p. 235-260.

Silva, A. R; Rêgo, E. R.; Cecon, P. R. Tamanho da amostra para a caracterização morfológica de frutos de pimenta. Horticultura Brasileira, v. 29, n. 1, p. 125-129, 2011. https://doi.org/10.1590/S010205362011000100015

SUDENE - Superintendência de Desenvolvimento do Nordeste. Dados pluviométricos mensais do Nordeste: Estado da Paraíba. 
Recife: SUDENE, 1990. (Série Pluviométrica, 5).

Sudré, C. P.; Rodrigues, R.; Riva, E. M.; Karasawa, M.; Amaral Júnior, A. T. Divergência genética entre acessos de pimentas e pimentões utilizando técnicas multivariadas. Horticultura Brasileira, v. 23, n. 1, p. 22-27, 2005. https://doi.org/ $10.1590 /$ S0102-05362005000100005

CC Informação da Licença: Este é um artigo Open Access distribuído sob os termos da Licença Creative Commons Attribution, que permite uso irrestrito, distribuição e reprodução em qualquer meio, desde que a obra original seja devidamente citada. 